\title{
Nehari-Type Ground State Positive Solutions for Superlinear Asymptotically Periodic Schrödinger Equations
}

\author{
Xiaoyan Lin $^{1}$ and X. H. Tang ${ }^{2}$ \\ ${ }^{1}$ Department of Mathematics, Huaihua College, Huaihua, Hunan 418008, China \\ ${ }^{2}$ School of Mathematics and Statistics, Central South University, Changsha, Hunan 410083, China \\ Correspondence should be addressed to Xiaoyan Lin; xiaoyanlin98@hotmail.com \\ Received 3 January 2014; Revised 23 May 2014; Accepted 11 June 2014; Published 29 June 2014 \\ Academic Editor: Marco Squassina
}

Copyright (c) $2014 \mathrm{X}$. Lin and X. H. Tang. This is an open access article distributed under the Creative Commons Attribution License, which permits unrestricted use, distribution, and reproduction in any medium, provided the original work is properly cited.

We deal with the existence of Nehari-type ground state positive solutions for the nonlinear Schrödinger equation $-\Delta u+V(x) u=$ $f(x, u), x \in \mathbb{R}^{N}, u \in H^{1}\left(\mathbb{R}^{N}\right)$. Under a weaker Nehari condition, we establish some existence criteria to guarantee that the above problem has Nehari-type ground state solutions by using a more direct method in two cases: the periodic case and the asymptotically periodic case.

\section{Introduction}

Consider the following semilinear Schrödinger equation:

$$
\begin{gathered}
-\Delta u+V(x) u=f(x, u), \quad x \in \mathbb{R}^{N}, \\
u \in H^{1}\left(\mathbb{R}^{N}\right),
\end{gathered}
$$

where $V: \mathbb{R}^{N} \rightarrow \mathbb{R}$ and $f: \mathbb{R}^{N} \times \mathbb{R} \rightarrow \mathbb{R}$.

The Schrödinger equation has found a great deal of interest last years because not only it is important in applications but also it provides a good model for developing mathematical methods. Many authors have studied the existence of entire solutions of Schrödinger equations under various stipulations (cf., e.g., [1-28] and the references quoted in them).

When $\inf _{\mathbb{R}^{N}} V(x)>0$ and $V(x)$ is periodic, Li et al. [12] made use of a combination of the techniques in $[13,14]$ with applications of Lions' concentration compactness principle $[26,29,30]$ to establish the following theorem.

Theorem 1 (see [12]). Assume that $V$ and $f$ satisfy the following assumptions:

(V0) $V \in C\left(\mathbb{R}^{N}, \mathbb{R}\right)$ and $\inf _{\mathbb{R}^{N}} V(x)>0 ;$

(V1) $V(x)$ is 1-periodic in each of $x_{1}, x_{2}, \ldots, x_{N}$;
(S0) $f \in C^{1}, f_{t}^{\prime}$ is a Caratheodory function, and there exists a constant $C>0$ such that

$$
\left|f_{t}^{\prime}(x, t)\right| \leq C\left(1+|t|^{2^{*}-2}\right), \quad \lim _{|t| \rightarrow \infty} \frac{\left|f_{t}^{\prime}(x, t)\right|}{|t|^{2^{*}-2}}=0
$$

(S1) $f(x, t)$ is 1-periodic in each of $x_{1}, x_{2}, \ldots, x_{N}$;

(S2) $f(x, t)=o(|t|)$, as $|t| \rightarrow 0$, uniformly in $x \in \mathbb{R}^{N}$;

(S3) $\lim _{|t| \rightarrow \infty}\left(|F(x, t)| /|t|^{2}\right)=\infty$, uniformly in $x \in \mathbb{R}^{N}$;

(S4) $f(x, t) /|t|$ is strictly increasing in $t$ on $\mathbb{R} \backslash\{0\}$ for every $x \in \mathbb{R}^{N}$.

Then problem (1) has a solution $u_{0} \in H^{1}\left(\mathbb{R}^{N}\right)$ such that $\Phi\left(u_{0}\right)=\inf _{\mathcal{N}} \Phi>0$, where

$$
\begin{aligned}
\Phi(u)= & \frac{1}{2} \int_{\mathbb{R}^{N}}\left(|\nabla u|^{2}+V(x) u^{2}\right) \mathrm{d} x \\
& -\int_{\mathbb{R}^{N}} F(x, u) \mathrm{d} x, \quad \forall u \in H^{1}\left(\mathbb{R}^{N}\right), \\
\left\langle\Phi^{\prime}(u), v\right\rangle= & \int_{\mathbb{R}^{N}}(\nabla u \cdot \nabla v+V(x) u v) \mathrm{d} x \\
& -\int_{\mathbb{R}^{N}} f(x, u) v \mathrm{~d} x, \quad \forall u, v \in H^{1}\left(\mathbb{R}^{N}\right)
\end{aligned}
$$


with $F(x, t):=\int_{0}^{t} f(x, s) \mathrm{d} s$, and

$$
\mathcal{N}=\left\{u \in H^{1}\left(\mathbb{R}^{N}\right):\left\langle\Phi^{\prime}(u), u\right\rangle=0, u \neq 0\right\} .
$$

The set $\mathcal{N}$ is the Nehari manifold, which contains infinitely many elements of $H^{1}\left(\mathbb{R}^{N}\right)$. In fact, for any $u \in$ $H^{1}\left(\mathbb{R}^{N}\right) \backslash\{0\}$, there exists $t=t(u)>0$ such that $t u \in \mathscr{N}$; see Lemma 9. Since $u_{0}$ is a solution at which $\Phi$ has least "energy" in set $\mathcal{N}$, we will call it a Nehari-type ground state solution.

We must point out that "the least energy solution" (which is sometimes also called the ground state solution in some references) is in fact a nontrivial solution $u_{0}$ which satisfies $\Phi\left(u_{0}\right)=\inf _{\mathscr{M}} \Phi$, where

$$
\mathscr{M}=\left\{u \in H^{1}\left(\mathbb{R}^{N}\right) \backslash\{0\}: \Phi^{\prime}(u)=0\right\}
$$

is a very small subset of $\mathcal{N}$; it may contain only one element. In general, it is much more difficult to find a solution $u_{0}$ for problem (1) with a constraint condition $\Phi\left(u_{0}\right)=\inf _{\mathcal{N}} \Phi$ than with one $\Phi\left(u_{0}\right)=\inf _{\mathscr{M}} \Phi$.

To establish the existence of Nehari-type ground state solutions, the so-called Nehari-type condition (S4) seems to be always necessary in the proof of the existence of ground states solutions for problem (1).

In recent paper $[15,20]$, Theorem 1 has been extended to the case where 0 is in the gap of the spectrum $\sigma(-\Delta+V)$, but an additional assumption on the nonlinearity $f$ is assumed in [15].

Motivated by $[12,17,20]$, in the present paper, we will develop a more direct method to generalize Theorem 1 by relaxing assumptions ( $\mathrm{V} 0),(\mathrm{S} 3)$, and (S4) in two cases: the periodic case and the asymptotically periodic case.

In the periodic case, we establish the following two theorems.

Theorem 2. Assume that $V$ and $f$ satisfy (V0), (V1), (S1), (S2), and the following assumptions:

$\left(\mathrm{S}^{\prime}\right) f \in C\left(\mathbb{R}^{N} \times \mathbb{R}, \mathbb{R}\right)$, and there exists a constant $C>0$ such that

$$
|f(x, t)| \leq C\left(1+|t|^{2^{*}-1}\right), \quad \lim _{|t| \rightarrow \infty} \frac{|f(x, t)|}{|t|^{2^{*}-1}}=0,
$$

uniformly in $x \in \mathbb{R}^{N}$;

$\left(\mathrm{S} 3^{\prime}\right) \lim _{|t| \rightarrow \infty}\left(|F(x, t)| /|t|^{2}\right)=\infty$, a.e. $x \in \mathbb{R}^{N} ;$

$\left(\mathrm{S} 4^{\prime}\right) f(x, t) /|t|$ is nondecreasing in $t$ on $\mathbb{R} \backslash\{0\}$ for every $x \in \mathbb{R}^{N}$

Then problem (1) has a solution $u_{0} \in H^{1}\left(\mathbb{R}^{N}\right)$ such that $\Phi\left(u_{0}\right)=\inf _{\mathscr{N}} \Phi>0$.

Theorem 3. Assume that $V$ and $f$ satisfy (V0), (V1), $\left(\mathrm{SO}^{\prime}\right)$, (S1), (S2), (S3'), and $\left(S 4^{\prime}\right)$. Then problem (1) has a positive solution $\bar{u} \in H^{1}\left(\mathbb{R}^{N}\right)$ such that $\Phi_{+}(\bar{u})=\inf _{\mathcal{N}_{+}} \Phi_{+}>0$, where

$$
\begin{array}{r}
\Phi_{+}(u)=\frac{1}{2} \int_{\mathbb{R}^{N}}\left(|\nabla u|^{2}+V(x) u^{2}\right) \mathrm{d} x-\int_{\mathbb{R}^{N}} F\left(x, u^{+}\right) \mathrm{d} x, \\
\forall u \in H^{1}\left(\mathbb{R}^{N}\right)
\end{array}
$$

with $u^{+}(x)=\max \{u(x), 0\}$ and $u^{-}(x)=\max \{-u(x), 0\}$, and

$$
\mathcal{N}_{+}=\left\{u \in H^{1}\left(\mathbb{R}^{N}\right):\left\langle\Phi_{+}^{\prime}(u), u\right\rangle=0, u \neq 0\right\} .
$$

In the asymptotically periodic case, $V(x)$ is allowed to be negative in some bounded domain in $\mathbb{R}^{N}$. Precisely, we use the following condition instead of (V0).

$\left(\mathrm{V} 0^{\prime}\right) V \in C\left(\mathbb{R}^{N}\right) \cap L^{\infty}\left(\mathbb{R}^{N}\right), V(x) \leq \bar{V} \in(0, \infty)$ for all $x \in \mathbb{R}^{N}$ and there exists a constant $\zeta_{0}>0$ such that

$$
\begin{aligned}
& \int_{\mathbb{R}^{N}}\left(|\nabla u|^{2}+V(x) u^{2}\right) \mathrm{d} x \\
& \quad \geq \zeta_{0} \int_{\mathbb{R}^{N}}[\bar{V}-V(x)]|u|^{2} \mathrm{~d} x, \quad \forall u \in H^{1}\left(\mathbb{R}^{N}\right) .
\end{aligned}
$$

$\left(\mathrm{V0}^{\prime}\right)$ Was first introduced by Deng et al. [6]; it is satisfied if the following assumption holds (see Lemma 7):

$\left(\mathrm{V0}^{\prime \prime}\right) V \in C\left(\mathbb{R}^{N}\right) \cap L^{\infty}\left(\mathbb{R}^{N}\right)$, and there exist two constants $a_{1}, a_{2}>0$ and a bounded measurable set $\Omega \subset \mathbb{R}^{N}$ such that $\left(a_{1}+a_{2}\right)|\Omega|^{2 / N} \leq S$, and

$$
V(x) \geq \begin{cases}-a_{1}, & x \in \Omega, \\ a_{2}, & x \notin \Omega,\end{cases}
$$

where $S=\inf _{u \in \mathscr{D}^{1,2}\left(\mathbb{R}^{N}\right),\|u\|_{2^{*}=1}\|\nabla u\|_{2}^{2}}$.

In this case, we establish the following two theorems.

Theorem 4. Assume that $V$ and $f$ satisfy $\left(V 0^{\prime}\right),\left(S 0^{\prime}\right),(S 2)$, (S3), $\left(S 4^{\prime}\right)$, and the following assumptions:

(V2) $V(x)=V_{0}(x)+V_{1}(x), V_{0} \in C\left(\mathbb{R}^{N},(0,+\infty)\right), V_{0}(x)$ is 1-periodic in each of $x_{1}, x_{2}, \ldots, x_{N}, V_{1}(x)<0$ for $|x|<1+\sqrt{N}$, and $V_{1}(x) \leq 0$ for $|x| \geq 1+\sqrt{N}$, $\lim _{|x| \rightarrow \infty}\left|V_{1}(x)\right|=0$;

$\left(\mathrm{S}^{\prime}\right) f(x, t)=f_{0}(x, t)+f_{1}(x, t) ; f_{0} \in C\left(\mathbb{R}^{N} \times \mathbb{R}, \mathbb{R}\right)$ satisfies (S1), (S2), and $\left(S 4^{\prime}\right) ; f_{1} \in C\left(\mathbb{R}^{N} \times \mathbb{R}, \mathbb{R}\right)$ satisfies that

$$
0 \leq t f_{1}(x, t) \leq a(x)\left(t^{2}+|t|^{p_{0}}\right), \quad \forall(x, t) \in \mathbb{R}^{N} \times \mathbb{R}
$$

where $p_{0} \in\left(2,2^{*}\right)$ and $a \in C\left(\mathbb{R}^{N}, \mathbb{R}^{+}\right)$with $\lim _{|x| \rightarrow \infty} a(x)=0$.

Then problem (1) has a solution $u_{0} \in H^{1}\left(\mathbb{R}^{N}\right)$ such that $\Phi\left(u_{0}\right)=\inf _{\mathscr{N}} \Phi>0$.

Theorem 5. Assume that $V$ and $f$ satisfy $\left(V 0^{\prime}\right),(V 2),\left(S 0^{\prime}\right)$, $\left(S 1^{\prime}\right),(S 2),(S 3)$, and $\left(S 4^{\prime}\right)$. Then problem (1) has a positive solution $\bar{u} \in H^{1}\left(\mathbb{R}^{N}\right)$ such that $\Phi_{+}(\bar{u})=\inf _{\mathcal{N}_{+}} \Phi_{+}>0$.

In our theorems, we give a new condition $\left(\mathrm{S} 4^{\prime}\right)$ which weakens Nehari-type condition (S3) considerably. 


\section{Preliminaries}

Lemma 6 (see [6, Lemma 2.3]). Assume that $V$ satisfies $\left(V 0^{\prime}\right)$. Then there exist two positive constants $C_{1}, C_{2}>0$ such that

$$
\begin{aligned}
C_{1}\|u\|_{H^{1}\left(\mathbb{R}^{N}\right)}^{2} & \leq \int_{\mathbb{R}^{N}}\left[|\nabla u|^{2}+V(x) u^{2}\right] \mathrm{d} x \\
& \leq C_{2}\|u\|_{H^{1}\left(\mathbb{R}^{N}\right)}^{2}, \quad \forall u \in H^{1}\left(\mathbb{R}^{N}\right),
\end{aligned}
$$

where $\|u\|_{H^{1}\left(\mathbb{R}^{N}\right)}$ is the usual norm of $H^{1}\left(\mathbb{R}^{N}\right)$

$$
\|u\|_{H^{1}\left(\mathbb{R}^{N}\right)}=\left[\int_{\mathbb{R}^{N}}\left(|\nabla u|^{2}+u^{2}\right) \mathrm{d} x\right]^{1 / 2}, \quad u \in H^{1}\left(\mathbb{R}^{N}\right) .
$$

By Lemma 6, we define an inner product

$$
(u, v)=\int_{\mathbb{R}^{N}}[(\nabla u \cdot \nabla v)+V(x) u v] \mathrm{d} x, \quad u, v \in H^{1}\left(\mathbb{R}^{N}\right),
$$

associated with the norm

$$
\|u\|=\left\{\int_{\mathbb{R}^{N}}\left[|\nabla u|^{2}+V(x) u^{2}\right] \mathrm{d} x\right\}^{1 / 2}, \quad u \in H^{1}\left(\mathbb{R}^{N}\right) .
$$

Then $H^{1}\left(\mathbb{R}^{N}\right)$ is a Hilbert space with this inner product. Moreover, under assumptions $\left(\mathrm{V}^{\prime}\right)$ and $\left(\mathrm{SO}^{\prime}\right)$, the functional $\Phi$ defined by (3) is of class $C^{1}\left(H^{1}\left(\mathbb{R}^{N}\right), \mathbb{R}\right)$.

Lemma 7. If $\left(V^{\prime \prime}\right)$ holds, then $\left(V 0^{\prime}\right)$ does.

Proof. By virtue of $\left(\mathrm{VO}^{\prime \prime}\right)$, the Hölder inequality, and the Sobolev inequality, we have

$$
\begin{aligned}
& \int_{\mathbb{R}^{N}}\left[|\nabla u|^{2}+V(x) u^{2}\right] \mathrm{d} x \\
& \geq \int_{\mathbb{R}^{N}}|\nabla u|^{2} \mathrm{~d} x-\left(a_{1}+a_{2}\right) \int_{\Omega} u^{2} \mathrm{~d} x+a_{2} \int_{\mathbb{R}^{N}} u^{2} \mathrm{~d} x \\
& \geq \int_{\mathbb{R}^{N}}|\nabla u|^{2} \mathrm{~d} x-\left(a_{1}+a_{2}\right)|\Omega|^{2 / N}\|u\|_{2^{*}}^{2}+a_{2} \int_{\mathbb{R}^{N}} u^{2} \mathrm{~d} x \\
& \geq\|\nabla u\|_{2}^{2}-\left(a_{1}+a_{2}\right)|\Omega|^{2 / N} S^{-1}\|\nabla u\|_{2}^{2}+a_{2} \int_{\mathbb{R}^{N}} u^{2} \mathrm{~d} x \\
& \geq a_{2} \int_{\mathbb{R}^{N}} u^{2} \mathrm{~d} x \\
& \geq \frac{a_{2}}{\bar{V}+a_{1}} \int_{\mathbb{R}^{N}}[\bar{V}-V(x)] u^{2} \mathrm{~d} x, \quad \forall u \in H^{1}\left(\mathbb{R}^{N}\right) .
\end{aligned}
$$

This shows that $\left(\mathrm{V}^{\prime}\right)$ holds with $\zeta_{0}=a_{2} /\left(\bar{V}+a_{1}\right)$.

Lemma 8. Let $X$ be a Banach space. Let $M_{0}$ be a closed subspace of the metric space $M$ and $\Gamma_{0} \subset C\left(M_{0}, X\right)$. Define

$$
\Gamma=\left\{\gamma \in C(M, X):\left.\gamma\right|_{M_{0}} \in \Gamma_{0}\right\} .
$$

If $\Psi \in C^{1}(X, \mathbb{R})$ satisfies

$$
\infty>c:=\inf _{\gamma \in \Gamma} \sup _{t \in M} \Psi(\gamma(t))>a:=\sup _{\gamma_{0} \in \Gamma_{0}} \sup _{t \in M_{0}} \Psi\left(\gamma_{0}(t)\right),
$$

then there exists a sequence $\left\{u_{n}\right\} \subset X$ satisfying

$$
\Psi\left(u_{n}\right) \longrightarrow c, \quad\left\|\Psi^{\prime}\left(u_{n}\right)\right\|\left(1+\left\|u_{n}\right\|\right) \longrightarrow 0 .
$$

Proof. For any $\gamma \in \Gamma$, define set $K_{\gamma}=\{\gamma(t): t \in M\}$ in $X$ and the collection $\mathscr{K}=\left\{K_{\gamma}: \gamma \in \Gamma\right\}$. Let $A=\left\{\gamma_{0}(t): \gamma_{0} \in \Gamma_{0}, t \in\right.$ $\left.M_{0}\right\}$,

$$
\begin{aligned}
& \Lambda=\left\{\varphi \in C(X, X): \varphi^{-1} \in C(X, X),\right. \\
& \quad \text { both } \varphi \text { and } \varphi^{-1} \text { are } \\
& \quad \text { bounded on bounded sets }\}, \\
& \Lambda(A)=\{\varphi \in \Lambda: \varphi(u)=u, u \in A\} .
\end{aligned}
$$

For any $\gamma \in \Gamma$ and $\varphi \in \Lambda(A)$, let $\gamma_{0}=\left.\gamma\right|_{M_{0}}$ and $\tilde{\gamma}(t)=$ $\varphi(\gamma(t)), t \in M$. Then $\gamma_{0} \in \Gamma_{0}$ and $\tilde{\gamma} \in C(M, X)$. Hence,

$$
\tilde{\gamma}(t)=\varphi\left(\gamma_{0}(t)\right)=\gamma_{0}(t), \quad \forall t \in M_{0} ;
$$

that is, $\left.\tilde{\gamma}\right|_{M_{0}}=\gamma_{0} \in \Gamma_{0}$. Therefore,

$$
\varphi(K) \in \mathscr{K}, \quad \forall \varphi \in \Lambda(A), K \in \mathscr{K} .
$$

These show that the collection $\mathscr{K}$ is a minimax system for $A$. Since (19) implies

$$
\infty>c:=\inf _{K \in \mathscr{K}} \sup _{K} \Psi>a:=\sup _{A} \Psi,
$$

it follows from Theorem 2.4 in [18] that the result is true.

Lemma 9. Under assumptions $\left(V 0^{\prime}\right),\left(S 0^{\prime}\right),(S 2)$, and $\left(S 3^{\prime}\right)$, for any $u \in H^{1}\left(\mathbb{R}^{N}\right) \backslash\{0\}$, there exists $t(u)>0$ such that $t(u) u \in$ $\mathcal{N}$.

Proof. Let $u \in H^{1}\left(\mathbb{R}^{N}\right) \backslash\{0\}$ be fixed and define the function $g(t):=\Phi(t u)$ on $[0, \infty)$. Clearly we have

$$
g^{\prime}(t)=0 \Longleftrightarrow t u \in \mathcal{N} \Longleftrightarrow\|u\|^{2}=\frac{1}{t} \int_{\mathbb{R}^{N}} f(x, t u) u \mathrm{~d} x .
$$

It is easy to verify, using (S2) and $\left(\mathrm{S}^{\prime}\right)$, that $g(0)=0$, $g(t)>0$ for $t>0$ small and $g(t)<0$ for $t$ large. Therefore $\max _{t \in[0, \infty)} g(t)$ is achieved at a $t_{0}=t(u)$ so that $g^{\prime}\left(t_{0}\right)=0$ and $t(u) u \in \mathcal{N}$.

Lemma 10. Under assumptions $\left(\mathrm{VO}^{\prime}\right),\left(S 0^{\prime}\right)$, and $\left(S 4^{\prime}\right)$,

$$
\begin{array}{r}
\Phi(u) \geq \Phi(t u)+\frac{1-t^{2}}{2}\left\langle\Phi^{\prime}(u), u\right\rangle, \\
\forall u \in H^{1}\left(\mathbb{R}^{N}\right), \quad t \geq 0 .
\end{array}
$$


Proof. For $\tau \neq 0,\left(\mathrm{~S} 4^{\prime}\right)$ yields

$$
\begin{aligned}
& f(x, s) \leq \frac{f(x, \tau)}{|\tau|}|s|, \quad s \leq \tau ; \\
& f(x, s) \geq \frac{f(x, \tau)}{|\tau|}|s|, \quad s \geq \tau .
\end{aligned}
$$

It follows that

$$
\frac{1-t^{2}}{2} \tau f(x, \tau) \geq \int_{t \tau}^{\tau} f(x, s) \mathrm{d} s, \quad t \geq 0 .
$$

Note that

$$
\left\langle\Phi^{\prime}(u), u\right\rangle=\|u\|^{2}-\int_{\mathbb{R}^{N}} f(x, u) u \mathrm{~d} x .
$$

Thus, by (3), (28), and (29), one has

$$
\begin{aligned}
& \Phi(u)-\Phi(t u) \\
&=\frac{1-t^{2}}{2}\|u\|^{2}+\int_{\mathbb{R}^{N}}[F(x, t u)-F(x, u)] \mathrm{d} x \\
&=\frac{1-t^{2}}{2}\left\langle\Phi^{\prime}(u), u\right\rangle \\
&+\int_{\mathbb{R}^{N}}\left[\frac{1-t^{2}}{2} f(x, u) u+F(x, t u)-F(x, u)\right] \mathrm{d} x \\
&=\frac{1-t^{2}}{2}\left\langle\Phi^{\prime}(u), u\right\rangle \\
& \quad+\int_{\mathbb{R}^{N}}\left[\frac{1-t^{2}}{2} f(x, u) u-\int_{t u}^{u} f(x, s) \mathrm{d} s\right] \mathrm{d} x \\
& \geq \frac{1-t^{2}}{2}\left\langle\Phi^{\prime}(u), u\right\rangle, \quad t \geq 0 .
\end{aligned}
$$

This shows that (26) holds.

Corollary 11. Under assumptions $\left(V 0^{\prime}\right),\left(S 0^{\prime}\right)$, and $\left(S 4^{\prime}\right)$, for $u \in \mathcal{N}$,

$$
\Phi(u) \geq \Phi(t u), \quad \forall t \geq 0
$$

We define

$$
\begin{gathered}
c_{1}:=\inf _{\mathcal{N}} \Phi, \quad c_{2}:=\inf _{u \in E, u \neq 0} \max _{t \geq 0} \Phi(t u), \\
c:=\inf _{\gamma \in \Gamma} \sup _{t \in[0,1]} \Phi(\gamma(t))
\end{gathered}
$$

where

$$
\Gamma=\{\gamma \in C([0,1], E): \gamma(0)=0, \Phi(\gamma(1))<0\} .
$$

Lemma 12. Under assumptions $\left(V 0^{\prime}\right),\left(S 0^{\prime}\right),(S 2),\left(S 3^{\prime}\right)$, and $\left(S 4^{\prime}\right)$, one has that $c_{1}=c_{2}=c>0$ and there exists a sequence $\left\{u_{n}\right\} \subset H^{1}\left(\mathbb{R}^{N}\right)$ satisfying

$$
\Phi\left(u_{n}\right) \longrightarrow c, \quad\left\|\Phi^{\prime}\left(u_{n}\right)\right\|\left(1+\left\|u_{n}\right\|\right) \longrightarrow 0 .
$$

Proof. (1) Both Lemma 9 and Corollary 11 imply that $c_{1}=c_{2}$. Next, we prove that $c=c_{1}=c_{2}$. By the definition of $c_{2}$, we choose a sequence $\left\{v_{n}\right\} \subset E \backslash\{0\}$ such that

$$
c_{2} \leq \max _{t \geq 0} \Phi\left(t v_{n}\right)<c_{2}+\frac{1}{n}, \quad n \in \mathbb{N} .
$$

Since $\Phi(t u)<0$ for $u \in E \backslash\{0\}$ and $t$ large, there exist $t_{n}=$ $t\left(v_{n}\right)>0$ and $s_{n}>t_{n}$ such that

$$
\Phi\left(t_{n} v_{n}\right)=\max _{t \geq 0} \Phi\left(t v_{n}\right), \quad \Phi\left(s_{n} v_{n}\right)<0, \quad n \in \mathbb{N} .
$$

Let $\gamma_{n}(t)=t s_{n} v_{n}$ for $t \in[0,1]$. Then $\gamma_{n} \in \Gamma$, and it follows from (35) and (36) that

$$
\sup _{t \in[0,1]} \Phi\left(\gamma_{n}(t)\right)=\max _{t \geq 0} \Phi\left(t v_{n}\right)<c_{2}+\frac{1}{n}, \quad n \in \mathbb{N},
$$

which implies that $c \leq c_{2}$. On the other hand, the manifold $\mathcal{N}$ separates $H^{1}\left(\mathbb{R}^{N}\right)$ into two components $E^{+}=\left\{u \in H^{1}\left(\mathbb{R}^{N}\right)\right.$ : $\left.\left\langle\Phi^{\prime}(u), u\right\rangle>0\right\} \cup\{0\}$ and $E^{-}=\left\{u \in H^{1}\left(\mathbb{R}^{N}\right):\left\langle\Phi^{\prime}(u), u\right\rangle<\right.$ $0\}$. By $\left(\mathrm{S} 4^{\prime}\right)$, one has

$$
f(x, t) t \geq 2 F(x, t), \quad \forall(x, t) \in \mathbb{R}^{N} \times \mathbb{R} .
$$

It follows that $\Phi(u) \geq 0$ for $u \in E^{+}$. By $\left(\mathrm{S}^{\prime}\right)$ and (S2), $E^{+}$ contains a small ball around the origin. Thus every $\gamma \in \Gamma$ has to cross $\mathcal{N}$, because $\gamma(0) \in E^{+}$and $\gamma(1) \in E^{-}$, and so $c_{1} \leq c$.

(2) In order to prove the second part of Lemma 12, we apply Lemma 8 with $M=[0,1], M_{0}=\{0,1\}$, and

$$
\Gamma_{0}=\left\{\gamma_{0}:\{0,1\} \longrightarrow H^{1}\left(\mathbb{R}^{N}\right): \gamma_{0}(0)=0, \Phi\left(\gamma_{0}(1)\right)<0\right\} .
$$

By $\left(\mathrm{S}^{\prime}\right)$ and (S2), there exists $r>0$ such that

$$
\min _{\|u\| \leq r} \Phi(u)=0, \quad \inf _{\|u\|=r} \Phi(u)>0 .
$$

Hence we obtain

$$
c \geq \inf _{\|u\|=r} \Phi(u)>0=\sup _{\gamma_{0} \in \Gamma_{0}} \sup _{t \in M_{0}} \Phi\left(\gamma_{0}(t)\right) .
$$

These show that all assumptions of Lemma 8 are satisfied. Therefore there exists a sequence $\left(u_{n}\right) \subset H^{1}\left(\mathbb{R}^{N}\right)$ satisfying (34).

Lemma 13. Under assumptions $\left(V 0^{\prime}\right),\left(S 0^{\prime}\right),(S 2),(S 3)$, and $\left(S 4^{\prime}\right)$, any sequence $\left\{u_{n}\right\} \subset H^{1}\left(\mathbb{R}^{N}\right)$ satisfying (34) is bounded in $H^{1}\left(\mathbb{R}^{N}\right)$.

Proof. To prove the boundedness of $\left\{u_{n}\right\}$, arguing by contradiction, suppose that $\left\|u_{n}\right\| \rightarrow \infty$. Let $v_{n}=u_{n} /\left\|u_{n}\right\|$. Then $\left\|v_{n}\right\|=1$. By Sobolev embedding theorem, there exists a constant $C_{3}>0$ such that

$$
\left\|v_{n}\right\|_{2}+\left\|v_{n}\right\|_{2^{*}} \leq C_{3} .
$$

Passing to a subsequence, we may assume that $v_{n} \rightarrow v$ in $H^{1}\left(\mathbb{R}^{N}\right), v_{n} \rightarrow v$ in $L_{\text {loc }}^{s}\left(\mathbb{R}^{N}\right), 2 \leq s<2^{*}$, and $v_{n} \rightarrow v$ a.e. on $\mathbb{R}^{N}$. 
If $\delta:=\limsup \sup _{n \rightarrow \infty} \sup _{y \in \mathbb{R}^{N}} \int_{B_{1}(y)}\left|v_{n}\right|^{2} \mathrm{~d} x=0$, then by Lions' concentration compactness principle [23, Lemma 1.21], $v_{n} \rightarrow 0$ in $L^{s}\left(\mathbb{R}^{N}\right)$ for $2<s<2^{*}$. Fix $p \in\left(2,2^{*}\right)$ and $R>$ $2 \sqrt{c}$. By $\left(\mathrm{S}^{\prime}\right)$ and $(\mathrm{S} 2)$, for $\varepsilon=c / 4\left[\left(R C_{3}\right)^{2}+\left(R C_{3}\right)^{2^{*}}\right]>0$ there exists $C_{\varepsilon}>0$ such that

$$
|F(x, u)| \leq \varepsilon\left(|u|^{2}+|u|^{2^{*}}\right)+C_{\varepsilon}|u|^{p} .
$$

It follows that

$$
\begin{aligned}
& \limsup _{n \rightarrow \infty} \int_{\mathbb{R}^{N}} F\left(x, R v_{n}\right) \mathrm{d} x \\
& \quad \leq \varepsilon\left[\left(R C_{3}\right)^{2}+\left(R C_{3}\right)^{2^{*}}\right]+R^{p} C_{\varepsilon_{n \rightarrow \infty}} \lim _{n \rightarrow v_{n}} \|_{p}^{p}=\frac{c}{4} .
\end{aligned}
$$

Hence, by using (34), (44), and Lemma 10, one has

$$
\begin{aligned}
c+o(1)= & \Phi\left(u_{n}\right) \\
\geq & \Phi\left(R v_{n}\right)+\left(\frac{1}{2}-\frac{R^{2}}{2\left\|u_{n}\right\|^{2}}\right)\left\langle\Phi^{\prime}\left(u_{n}\right), u_{n}\right\rangle \\
= & \frac{R^{2}}{2}-\int_{\mathbb{R}^{N}} F\left(x, R v_{n}\right) \mathrm{d} x \\
& +\left(\frac{1}{2}-\frac{R^{2}}{2\left\|u_{n}\right\|^{2}}\right)\left\langle\Phi^{\prime}\left(u_{n}\right), u_{n}\right\rangle \\
\geq & \frac{R^{2}}{2}-\frac{c}{4}+o(1)>\frac{7 c}{4}+o(1),
\end{aligned}
$$

which is a contradiction. Thus, $\delta>0$.

Going if necessary to a subsequence, we may assume the existence of $k_{n} \in \mathbb{Z}^{N}$ such that $\int_{B_{1+\sqrt{N}}\left(k_{n}\right)}\left|v_{n}\right|^{2} \mathrm{~d} x>\delta / 2$. Let $w_{n}(x)=v_{n}\left(x+k_{n}\right)$. Then it follows that

$$
\int_{B_{1+\sqrt{N}}(0)}\left|w_{n}\right|^{2} \mathrm{~d} x>\frac{\delta}{2} .
$$

Now we define $\tilde{u}_{n}(x)=u_{n}\left(x+k_{n}\right)$, then $\tilde{u}_{n} /\left\|u_{n}\right\|=w_{n}$, and $\left\|w_{n}\right\|_{H^{1}\left(\mathbb{R}^{N}\right)}=\left\|u_{n}\right\|_{H^{1}\left(\mathbb{R}^{N}\right)} /\left\|u_{n}\right\| \leq C_{4}$ for some $C_{4}>0$. Passing to a subsequence, we have $w_{n} \rightarrow w$ in $H^{1}\left(\mathbb{R}^{N}\right), w_{n} \rightarrow w$ in $L_{\text {loc }}^{s}\left(\mathbb{R}^{N}\right), 2 \leq s<2^{*}$, and $w_{n} \rightarrow w$ a.e. on $\mathbb{R}^{N}$. Thus, (46) implies that $w \neq 0$. Hence, it follows from (34), (S3), and Fatou's lemma that

$$
\begin{aligned}
0 & =\lim _{n \rightarrow \infty} \frac{c+o(1)}{\left\|u_{n}\right\|^{2}}=\lim _{n \rightarrow \infty} \frac{\Phi\left(u_{n}\right)}{\left\|u_{n}\right\|^{2}} \\
& =\lim _{n \rightarrow \infty}\left[\frac{1}{2}-\int_{\mathbb{R}^{N}} \frac{F\left(x+k_{n}, \tilde{u}_{n}\right)}{\widetilde{u}_{n}^{2}} w_{n}^{2} \mathrm{~d} x\right] \\
& \leq \frac{1}{2}-\liminf _{n \rightarrow \infty} \int_{\mathbb{R}^{N}} \frac{F\left(x+k_{n}, \widetilde{u}_{n}\right)}{\widetilde{u}_{n}^{2}} w_{n}^{2} \mathrm{~d} x \\
& \leq \frac{1}{2}-\int_{\mathbb{R}^{N}} \liminf _{n \rightarrow \infty} \frac{F\left(x+k_{n}, \tilde{u}_{n}\right)}{\widetilde{u}_{n}^{2}} w_{n}^{2} \mathrm{~d} x=-\infty,
\end{aligned}
$$

which is a contradiction. Thus $\left\{u_{n}\right\}$ is bounded in $H^{1}\left(\mathbb{R}^{N}\right)$.
Remark 14. In the proof of Lemma 13, (S3) is used only in (47). Hence, it can be weakened to $\left(\mathrm{S} 3^{\prime}\right)$ if $f(x, t)$ is 1-periodic in each of $x_{1}, x_{2}, \ldots, x_{N}$.

\section{The Proofs of Theorems}

Proof of Theorem 2. Lemma 12 implies the existence of a sequence $\left\{u_{n}\right\} \subset H^{1}\left(\mathbb{R}^{N}\right)$ satisfying (34), by a standard argument; we can prove Theorem 2 .

Proof of Theorem 3. In view of the proofs of Lemmas 12 and 13 , we can show that the conclusions of Lemmas 12 and 13 still hold if $\Phi$ and $\mathcal{N}$ are replaced by $\Phi_{+}$and $\mathcal{N}_{+}$, respectively. Hence, there exists a bounded sequence $\left\{u_{n}\right\} \subset H^{1}\left(\mathbb{R}^{N}\right)$ satisfying

$$
\Phi_{+}\left(u_{n}\right) \longrightarrow c_{+}, \quad\left\|\Phi_{+}^{\prime}\left(u_{n}\right)\right\|\left(1+\left\|u_{n}\right\|\right) \longrightarrow 0
$$

where $c_{+}=\inf _{u \in \mathcal{N}_{+}} \Phi_{+}(u)$. The rest of the proof is standard, so we omit it.

To prove Theorems 4 and 5, we define functional $\Phi_{0}$ and $\Phi_{+, 0}$ as follows:

$$
\begin{array}{r}
\Phi_{0}(u)=\frac{1}{2} \int_{\mathbb{R}^{N}}\left(|\nabla u|^{2}+V_{0}(x) u^{2}\right) \mathrm{d} x-\int_{\mathbb{R}^{N}} F_{0}(x, u) \mathrm{d} x \\
u \in H^{1}\left(\mathbb{R}^{N}\right)
\end{array}
$$

$$
\begin{array}{r}
\Phi_{+, 0}(u)=\frac{1}{2} \int_{\mathbb{R}^{N}}\left(|\nabla u|^{2}+V_{0}(x) u^{2}\right) \mathrm{d} x-\int_{\mathbb{R}^{N}} F_{0}\left(x, u^{+}\right) \mathrm{d} x \\
u \in H^{1}\left(\mathbb{R}^{N}\right),
\end{array}
$$

where $F_{0}(x, t):=\int_{0}^{t} f_{0}(x, s) \mathrm{d} s$. Then (V2), $\left(\mathrm{S}^{\prime}\right)$, and $\left(\mathrm{S}^{\prime}{ }^{\prime}\right)$ imply that $\Phi_{0} \in C^{1}\left(H^{1}\left(\mathbb{R}^{N}\right), \mathbb{R}\right)$ and

$$
\begin{aligned}
\left\langle\Phi_{0}^{\prime}(u), v\right\rangle= & \int_{\mathbb{R}^{N}}\left(\nabla u \nabla v+V_{0}(x) u v\right) \mathrm{d} x \\
& -\int_{\mathbb{R}^{N}} f_{0}(x, u) v \mathrm{~d} x, \quad u, v \in H^{1}\left(\mathbb{R}^{N}\right) .
\end{aligned}
$$

Proof of Theorem 4. Lemma 12 implies the existence of a sequence $\left\{u_{n}\right\} \subset H^{1}\left(\mathbb{R}^{N}\right)$ satisfying (34). By Lemma 13, $\left\{u_{n}\right\}$ is bounded in $H^{1}\left(\mathbb{R}^{N}\right)$. Passing to a subsequence, we have $u_{n} \rightarrow u_{0}$ in $H^{1}\left(\mathbb{R}^{N}\right)$. Next, we prove $u_{0} \neq 0$.

Arguing by contradiction, suppose that $u_{0}=0$; that is, $u_{n} \rightarrow 0$ in $H^{1}\left(\mathbb{R}^{N}\right)$, and so $u_{n} \rightarrow 0$ in $L_{\text {loc }}^{s}\left(\mathbb{R}^{N}\right), 2 \leq s<2^{*}$, and $u_{n} \rightarrow 0$ a.e. on $\mathbb{R}^{N}$. For any $\varepsilon>0$, it follows from (V2) 
that there exists $R_{\varepsilon}>0$ such that $\left|V_{1}(x)\right| \leq \varepsilon$ for $|x| \geq R_{\varepsilon}$. Hence,

$$
\begin{aligned}
& \int_{\mathbb{R}^{N}}\left|V_{1}(x)\right| u_{n}^{2} \mathrm{~d} x \\
& \quad=\int_{B_{R_{\varepsilon}}(0)}\left|V_{1}(x)\right| u_{n}^{2} \mathrm{~d} x+\int_{\mathbb{R}^{N} \backslash B_{R_{\varepsilon}}(0)}\left|V_{1}(x)\right| u_{n}^{2} \mathrm{~d} x \\
& \quad \leq \sup _{\mathbb{R}^{N}}\left|V_{1}(x)\right| \int_{B_{R_{\varepsilon}}(0)} u_{n}^{2} \mathrm{~d} x+\varepsilon \int_{\mathbb{R}^{N} \backslash B_{R_{\varepsilon}}(0)} u_{n}^{2} \mathrm{~d} x \\
& \quad \leq o(1)+\varepsilon\left\|u_{n}\right\|_{2}^{2} \leq o(1)+C_{3} \varepsilon .
\end{aligned}
$$

Since $\varepsilon>0$ is arbitrary, we have

$$
\lim _{n \rightarrow \infty} \int_{\mathbb{R}^{N}} V_{1}(x) u_{n}^{2} \mathrm{~d} x=0 .
$$

Similarly, by $\left(S 1^{\prime}\right)$, one has

$$
\begin{gathered}
\lim _{n \rightarrow \infty} \int_{\mathbb{R}^{N}} F_{1}\left(x, u_{n}\right) \mathrm{d} x=0, \\
\lim _{n \rightarrow \infty} \int_{\mathbb{R}^{N}} f_{1}\left(x, u_{n}\right) u_{n} \mathrm{~d} x=0 .
\end{gathered}
$$

Note that

$$
\begin{aligned}
\Phi_{0}(u)= & \Phi(u)-\frac{1}{2} \int_{\mathbb{R}^{N}} V_{1}(x) u^{2} \mathrm{~d} x \\
& +\int_{\mathbb{R}^{N}} F_{1}(x, u) \mathrm{d} x, \quad \forall u \in H^{1}\left(\mathbb{R}^{N}\right), \\
\left\langle\Phi_{0}^{\prime}(u), v\right\rangle= & \left\langle\Phi^{\prime}(u), v\right\rangle-\int_{\mathbb{R}^{N}} V_{1}(x) u v \mathrm{~d} x \\
& +\int_{\mathbb{R}^{N}} f_{1}(x, u) v \mathrm{~d} x, \quad \forall u, v \in H^{1}\left(\mathbb{R}^{N}\right) .
\end{aligned}
$$

From (34) and (53)-(55), one has

$$
\Phi_{0}\left(u_{n}\right) \longrightarrow c, \quad\left\|\Phi_{0}^{\prime}\left(u_{n}\right)\right\|\left(1+\left\|u_{n}\right\|\right) \longrightarrow 0 .
$$

By a standard argument, we may prove that there exists $k_{n} \in \mathbb{Z}^{N}$, going if necessary to a subsequence, such that

$$
\int_{B_{1+\sqrt{N}}\left(k_{n}\right)}\left|u_{n}\right|^{2} \mathrm{~d} x>\frac{\delta}{2}>0 .
$$

Let $v_{n}(x)=u_{n}\left(x+k_{n}\right)$. Then $\left\|v_{n}\right\|_{H^{1}\left(\mathbb{R}^{N}\right)}=\left\|u_{n}\right\|_{H^{1}\left(\mathbb{R}^{N}\right)}$, and

$$
\int_{B_{1+\sqrt{N}}(0)}\left|v_{n}\right|^{2} \mathrm{~d} x>\frac{\delta}{2} .
$$

Since $V_{0}(x)$ and $f_{0}(x, u)$ are periodic, we have

$$
\Phi_{0}\left(v_{n}\right) \longrightarrow c, \quad\left\|\Phi_{0}^{\prime}\left(v_{n}\right)\right\|\left(1+\left\|v_{n}\right\|\right) \longrightarrow 0 .
$$

Since $\left\{v_{n}\right\}$ is bounded in $H^{1}\left(\mathbb{R}^{N}\right)$, passing to a subsequence, we have $v_{n} \rightarrow \bar{v}$ in $H^{1}\left(\mathbb{R}^{N}\right), v_{n} \rightarrow \bar{v}$ in $L_{\text {loc }}^{s}\left(\mathbb{R}^{N}\right), 2 \leq s<2^{*}$, and $v_{n} \rightarrow \bar{v}$ a.e. on $\mathbb{R}^{N}$. Obviously, (58) implies that $\bar{v}(x) \not \equiv 0$ for $x \in B_{1+\sqrt{N}}(0)$. By a standard argument, we can prove that $\Phi_{0}^{\prime}(\bar{v})=0$ and $\Phi_{0}(\bar{v}) \leq c$ by using $(59)$.

Since $\bar{v} \neq 0$, it follows from Lemma 9 that there exists $t_{0}=$ $t(\bar{v})$ such that $t_{0} \bar{v} \in \mathcal{N}$, and so $\Phi\left(t_{0} \bar{v}\right) \geq c$. On the other hand, from (49), (51), (V2), $\left(\mathrm{S}^{\prime}\right)$, and $\left(\mathrm{S} 4^{\prime}\right)$, we have

$$
\begin{aligned}
c \geq & \Phi_{0}(\bar{v}) \\
= & \Phi_{0}\left(t_{0} \bar{v}\right) \\
& \quad+\int_{\mathbb{R}^{N}}\left[\frac{1-t_{0}^{2}}{2} f_{0}(x, \bar{v}) \bar{v}+F_{0}\left(x, t_{0} \bar{v}\right)-F_{0}(x, \bar{v})\right] \mathrm{d} x \\
\geq & \Phi_{0}\left(t_{0} \bar{v}\right) \\
= & \Phi\left(t_{0} \bar{v}\right)-\frac{t_{0}^{2}}{2} \int_{\mathbb{R}^{N}} V_{1}(x) \bar{v}^{2} \mathrm{~d} x+\int_{\mathbb{R}^{N}} F_{1}\left(x, t_{0} \bar{v}\right) \mathrm{d} x \\
> & \Phi\left(t_{0} \bar{v}\right) \geq c .
\end{aligned}
$$

This contradiction implies that $u_{0} \neq 0$. By a standard argument, we can prove that $\Phi^{\prime}\left(u_{0}\right)=0$ and $\Phi\left(u_{0}\right)=c=\inf _{\mathcal{N}} \Phi$. This shows that $u_{0} \in H^{1}\left(\mathbb{R}^{N}\right)$ is a solution for problem (1) with $\Phi\left(u_{0}\right)=\inf _{\mathscr{N}} \Phi>0$.

Proof of Theorem 5. Similar to the proof of Theorem 3, there exists a bounded sequence $\left\{u_{n}\right\} \subset H^{1}\left(\mathbb{R}^{N}\right)$ satisfying (48). Passing to an appropriate subsequence, we have that $u_{n} \rightarrow \bar{u}$ in $H^{1}\left(\mathbb{R}^{N}\right)$. Next, we prove $\bar{u} \neq 0$.

Arguing by contradiction, suppose that $\bar{u}=0$; that is, $u_{n} \rightarrow 0$ in $H^{1}\left(\mathbb{R}^{N}\right)$. Then, $u_{n} \rightarrow 0$ in $L_{\text {loc }}^{s}\left(\mathbb{R}^{N}\right), 2 \leq$ $s<2^{*}$, and $u_{n} \rightarrow 0$ a.e. on $\mathbb{R}^{N}$. Analogous to the proof of Theorem 4, we can demonstrate that there exists a $\bar{v} \epsilon$ $H^{1}\left(\mathbb{R}^{N}\right) \backslash\{0\}$ with $\bar{v}(x) \not \equiv \quad 0$ for $x \in B_{1+\sqrt{N}}(0)$ such that $\Phi_{+, 0}^{\prime}(\bar{v})=0$ and $\Phi_{+, 0}(\bar{v}) \leq c$. By a standard argument, we can show that $\bar{v} \geq 0$.

Since $\bar{v} \geq(\not \equiv) 0$, it follows from Lemma 9 that there exists $t_{0}=t(\bar{v})$ such that $t_{0} \bar{v} \in \mathcal{N}_{+}$, and so $\Phi_{+}\left(t_{0} \bar{v}\right) \geq c$. On the other hand, from (49), (51), (V2), $\left(\mathrm{S1}^{\prime}\right)$, and $\left(\mathrm{S} 4^{\prime}\right)$, we have

$$
\begin{aligned}
& c \geq \Phi_{+, 0}(\bar{v}) \\
& =\Phi_{+, 0}\left(t_{0} \bar{v}\right)+\int_{\mathbb{R}^{N}}\left[\frac{1-t_{0}^{2}}{2} f_{0}(x, \bar{v}) \bar{v}\right. \\
& \left.+F_{0}\left(x, t_{0} \bar{v}\right)-F_{0}(x, \bar{v})\right] \mathrm{d} x \\
& \geq \Phi_{+, 0}\left(t_{0} \bar{v}\right) \\
& =\Phi_{+}\left(t_{0} \bar{v}\right)-\frac{t_{0}^{2}}{2} \int_{\mathbb{R}^{N}} V_{1}(x) \bar{v}^{2} \mathrm{~d} x+\int_{\mathbb{R}^{N}} F_{1}\left(x, t_{0} \bar{v}\right) \mathrm{d} x \\
& >\Phi_{+}\left(t_{0} \bar{v}\right) \geq c \text {. }
\end{aligned}
$$

This contradiction shows that $\bar{u} \neq 0$. In the same way as the last part of the proof of Theorem 1, we can deduce that $\Phi_{+}^{\prime}(\bar{u})=0$ and $\Phi_{+}(\bar{u})=c=\inf _{\mathcal{N}_{+}} \Phi_{+}$. By a standard 
argument, we can demonstrate that $\bar{u} \geq 0$. Therefore, $\bar{u} \in$ $H^{1}\left(\mathbb{R}^{N}\right)$ is a positive solution for problem (1) with $\Phi_{+}(\bar{u})=$ $\inf _{\mathcal{N}_{+}} \Phi_{+}>0$.

\section{Conflict of Interests}

The authors declare that there is no conflict of interests regarding the publication of this paper.

\section{Acknowledgment}

This work is partially supported by the NNSF (no. 11171351) of China.

\section{References}

[1] S. Alama and Y. Y. Li, "On "multibump" bound states for certain semilinear elliptic equations," Indiana University Mathematics Journal, vol. 41, no. 4, pp. 983-1026, 1992.

[2] S. Angenent, "The shadowing lemma for elliptic PDE," in Dynamics of Infinite Dimensional Systems, vol. 37 of NATO ASI Series, pp. 7-22, Springer, Berlin, Germany, 1986.

[3] T. Bartsch and Y. Ding, "Homoclinic solutions of an infinitedimensional Hamiltonian system," Mathematische Zeitschrift, vol. 240, no. 2, pp. 289-310, 2002.

[4] T. Bartsch, A. Pankov, and Z. Wang, "Nonlinear Schrödinger equations with steep potential well," Communications in Contemporary Mathematics, vol. 3, no. 4, pp. 549-569, 2001.

[5] J. Chabrowski and A. Szulkin, "On a semilinear Schrödinger equation with critical Sobolev exponent," Proceedings of the American Mathematical Society, vol. 130, no. 1, pp. 85-93, 2002.

[6] Y. Deng, L. Jin, and S. Peng, "Solutions of Schrödinger equations with inverse square potential and critical nonlinearity," Journal of Differential Equations, vol. 253, no. 5, pp. 1376-1398, 2012.

[7] Y. Ding and A. Szulkin, "Bound states for semilinear Schrödinger equations with sign-changing potential," Calculus of Variations and Partial Differential Equations, vol. 29, no. 3, pp. 397-419, 2007.

[8] Y. Ding and S. X. Luan, "Multiple solutions for a class of nonlinear Schrödinger equations," Journal of Differential Equations, vol. 207, no. 2, pp. 423-457, 2004.

[9] Y. Ding and C. Lee, "Multiple solutions of Schrödinger equations with indefinite linear part and super or asymptotically linear terms," Journal of Differential Equations, vol. 222, no. 1, pp. 137-163, 2006.

[10] W. Kryszewski and A. Szulkin, "Generalized linking theorem with an application to a semilinear Schrödinger equation," Advances in Differential Equations, vol. 3, no. 3, pp. 441-472, 1998.

[11] W. Kryszewski and A. Szulkin, "Infinite-dimensional homology and multibump solutions," Journal of Fixed Point Theory and Applications, vol. 5, no. 1, pp. 1-35, 2009.

[12] Y. Q. Li, Z. Q. Wang, and J. Zeng, "Ground states of nonlinear Schrödinger equations with potentials," Annales de l'Institut Henri Poincaré (C) Analyse Non Linéaire, vol. 23, no. 6, pp. 829837, 2006.

[13] J. Q. Liu, Y. Q. Wang, and Z. Q. Wang, "Solutions for quasilinear Schrödinger equations via the Nehari method," Communications in Partial Differential Equations, vol. 29, no. 5-6, pp. 879901, 2004.
[14] Z. Liu and Z.-Q. Wang, "On the Ambrosetti-Rabinowitz superlinear condition," Advanced Nonlinear Studies, vol. 4, pp. 561572, 2004.

[15] A. Pankov, "Periodic nonlinear Schrödinger equation with application to photonic crystals," Milan Journal of Mathematics, vol. 73, pp. 259-287, 2005.

[16] P. H. Rabinowitz, Minimax Methods in Critical Point Theory with Applications to Differential Equations, vol. 65 of Conference Board of the Mathematical Sciences Regional Conference Series in Mathematics, American Mathematical Society, Providence, RI, USA, 1986.

[17] P. H. Rabinowitz, "On a class of nonlinear Schrödinger equations," Zeitschrift für Angewandte Mathematik und Physik, vol. 43, no. 2, pp. 270-291, 1992.

[18] M. Schechter, Minimax Systems and Critical Point Theory, Birkhäuser, Boston, Mass, USA, 2009.

[19] M. Schechter and W. Zou, "Double linking theorem and multiple solutions," Journal of Functional Analysis, vol. 205, no. 1, pp. 37-61, 2003.

[20] A. Szulkin and T. Weth, "Ground state solutions for some indefinite variational problems," Journal of Functional Analysis, vol. 257, no. 12, pp. 3802-3822, 2009.

[21] X. H. Tang, "Infinitely many solutions for semilinear Schrödinger equations with sign-changing potential and nonlinearity," Journal of Mathematical Analysis and Applications, vol. 401, no. 1, pp. 407-415, 2013.

[22] X. H. Tang, "New super-quadratic conditions on ground state solutions for superlinear Schrödinger equation," Advanced Nonlinear Studies, vol. 14, pp. 349-361, 2014.

[23] X. H. Tang, "New conditions on nonlinearity for a periodic Schrödinger equation having zero as spectrum," Journal of Mathematical Analysis and Applications, vol. 413, no. 1, pp. 392410, 2014.

[24] C. Troestler and M. Willem, "Nontrivial solution of a semilinear Schrödinger equation," Communications in Partial Differential Equations, vol. 21, no. 9-10, pp. 1431-1449, 1996.

[25] F. A. van Heerden, "Homoclinic solutions for a semilinear elliptic equation with an asymptotically linear nonlinearity," Calculus of Variations and Partial Differential Equations, vol. 20, no. 4, pp. 431-455, 2004.

[26] M. Willem, Minimax Theorems, Birkhäuser, Boston, Mass, USA, 1996.

[27] M. Yang, "Ground state solutions for a periodic Schrödinger equation with superlinear nonlinearities," Nonlinear Analysis: Theory, Methods \& Applications, vol. 72, no. 5, pp. 2620-2627, 2010.

[28] M. Yang, W. Chen, and Y. Ding, "Solutions for periodic Schrödinger equation with spectrum zero and general superlinear nonlinearities," Journal of Mathematical Analysis and Applications, vol. 364, no. 2, pp. 404-413, 2010.

[29] P. L. Lions, "The concentration-compactness principle in the calculus of variations. The locally compact case. I," Annales de l'Institut Henri Poincaré. Analyse Non Linéaire, vol. 1, pp. 109145, 1984.

[30] M. Struwe, Variational Methods. Applications to Nonlinear Partial Differential Equations and Hamiltonian Systems, Springer, Berlin, Germany, 2000. 


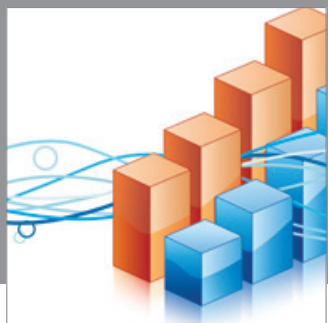

Advances in

Operations Research

mansans

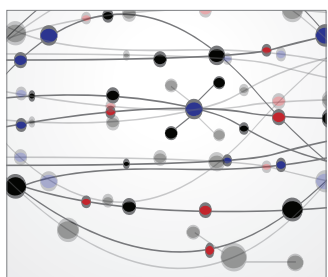

The Scientific World Journal
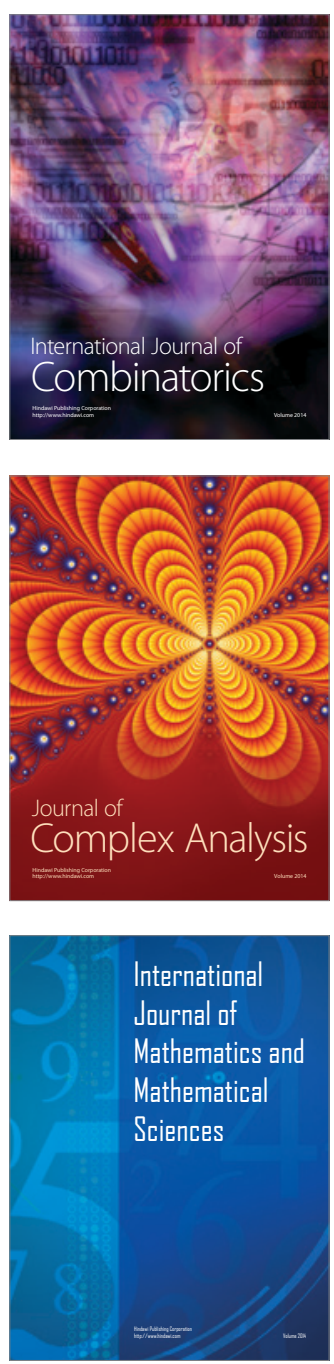
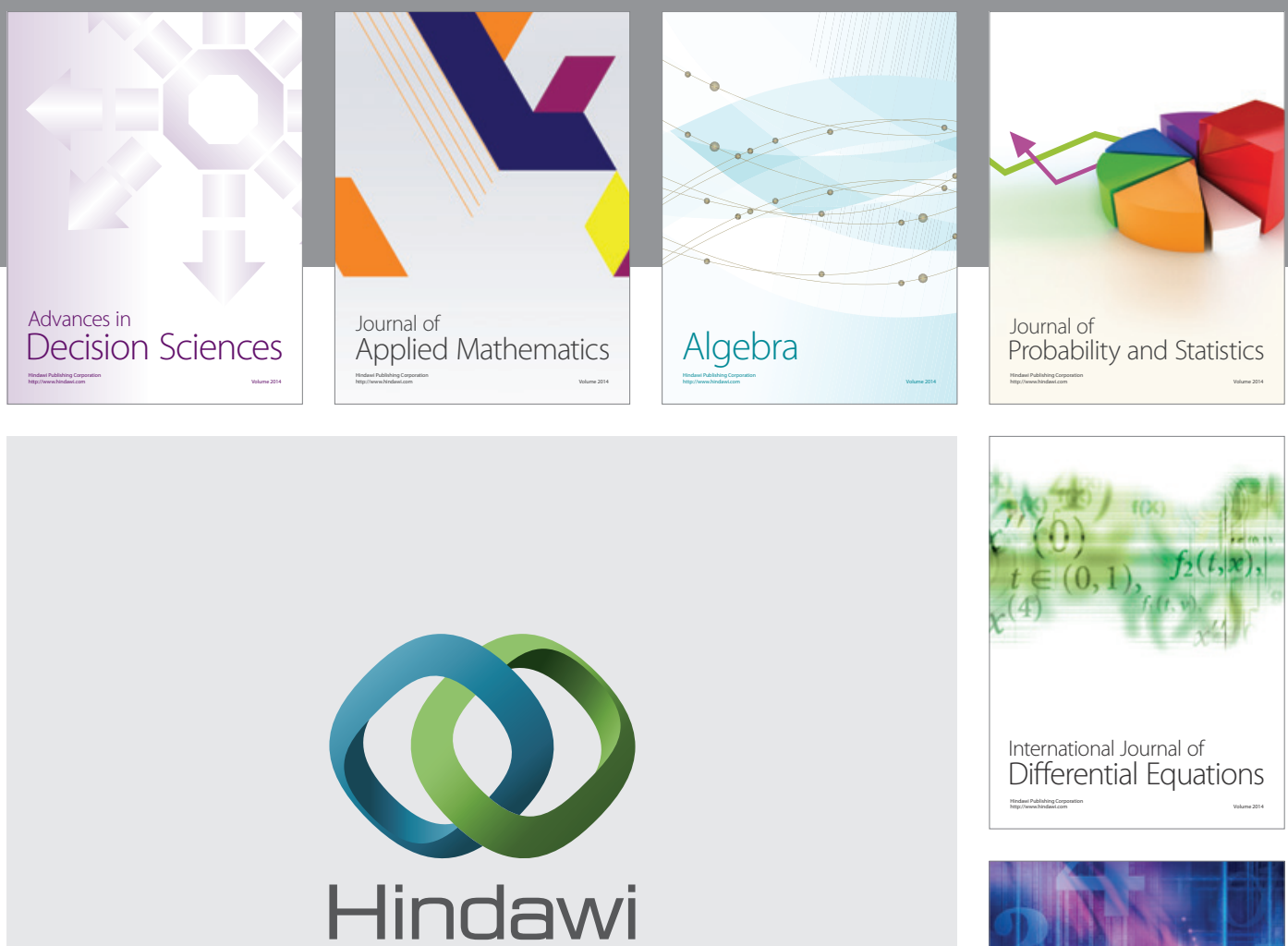

Submit your manuscripts at http://www.hindawi.com
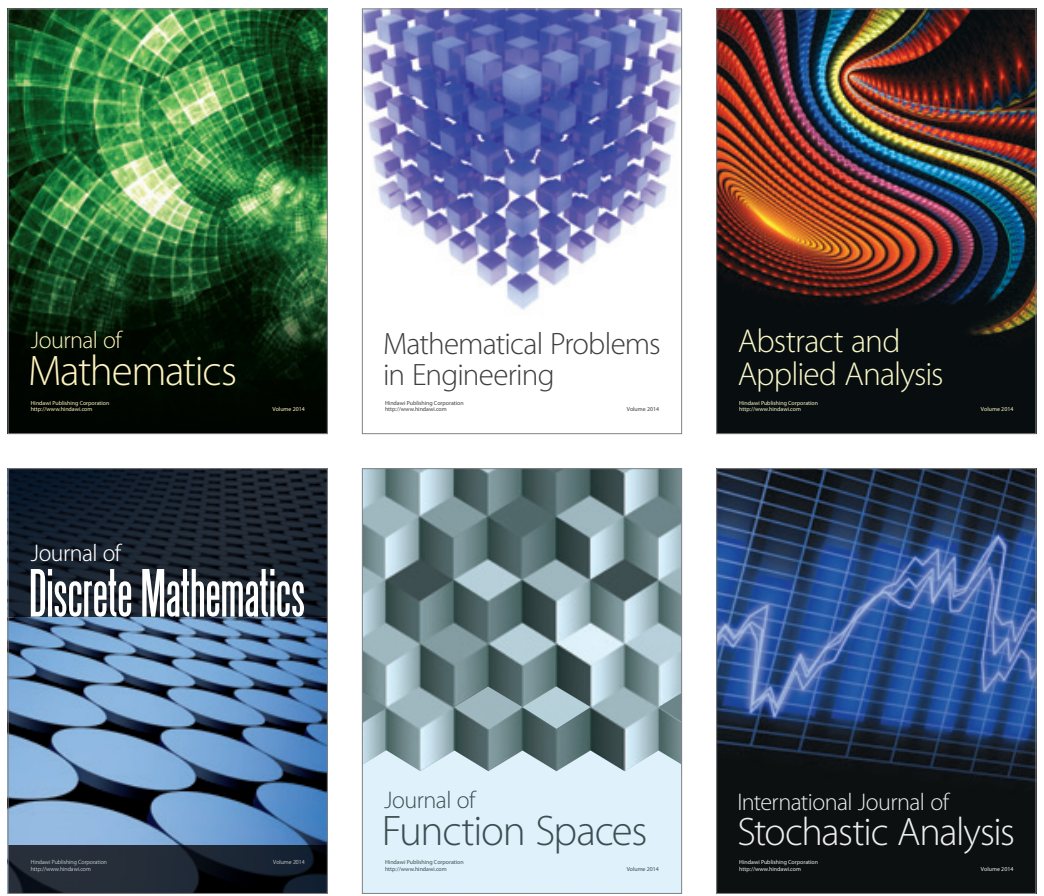

Journal of

Function Spaces

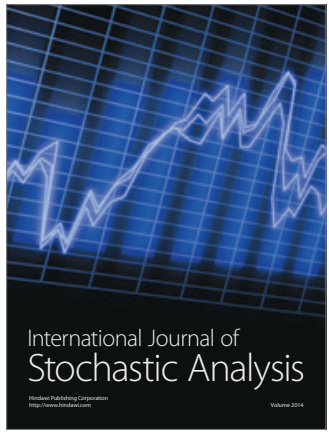

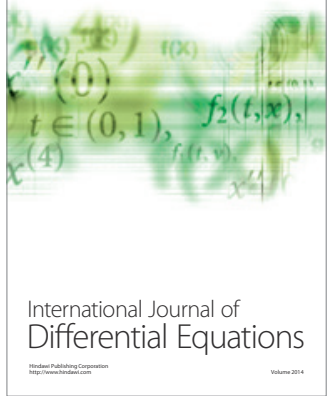
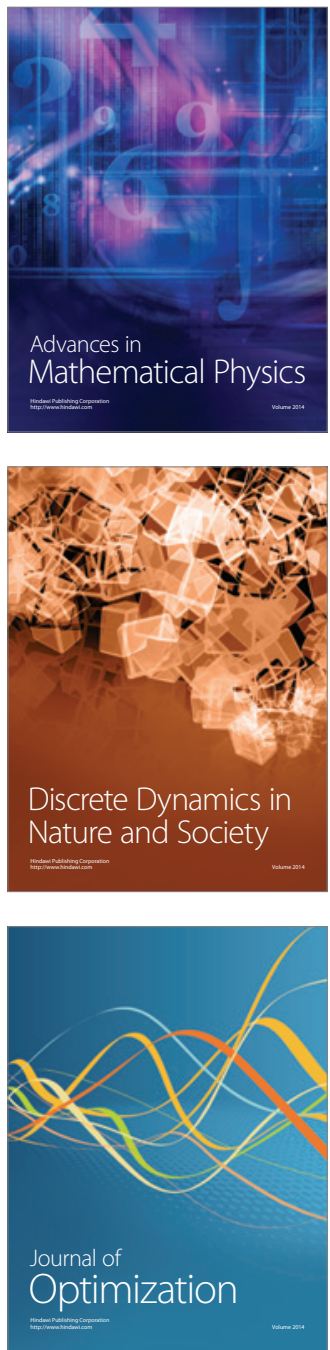